\title{
Stochastic Anti-Resonance in a Fibre Raman Amplifier
}

\author{
Vladimir L. Kalashnikov \\ Aston Institute of Photonic Technologies, \\ Aston University, Aston Triangle, Birmingham \\ B4 7ET, UK \\ v.kalashnikov@aston.ac.uk
}

\author{
Sergey V. Sergeyev \\ Aston Institute of Photonic Technologies, \\ Aston University, Aston Triangle, Birmingham \\ B4 7ET, UK \\ s.sergeyev@aston.ac.uk
}

\begin{abstract}
Stochastic anti-resonance, that is resonant enhancement of randomness caused by polarization mode beatings, is analyzed both numerically and analytically on an example of fibre Raman amplifier with randomly varying birefringence. As a result of such anti-resonance, the polarization mode dispersion growth causes an escape of the signal state of polarization from a metastable state corresponding to the pulling of the signal to the pump state of polarization.This phenomenon reveals itself in abrupt growth of gain fluctuations as well as in dropping of Hurst parameter and Kramers length characterizing long memory in a system and noise induced escape from the polarization pulling state. The results based on analytical multiscale averaging technique agree perfectly with the numerical data obtained by direct numerical simulations of underlying stochastic differential equations. This challenging outcome would allow replacing the cumbersome numerical simulations for real-world extra-long high-speed communication systems.
\end{abstract}

Keywords-stochastic anti-resonance; fibre Raman amplifier; telecommunication systems; multi-scaling; stochastic differential equations

\section{INTRODUCTION}

Most processes in nature are affected by noise leading to a complexity of dynamics. Noise cannot be considered as only disturbing factor because it can modify essentially phase space and evolution of a system. One has to note three main phenomena in this context: i) stochastic resonance, when a noise enhances system response to an external periodic perturbation, ii) stochastic anti-resonance, when an external periodic perturbation enhances a noise action, and iii) coherence resonance, when a noise creates some new coherent-like states in a system $[1,2]$.

In this work we present the results of extensive numerical simulations of a fibre Raman amplifier with inherent stochastic birefringence, which demonstrates the resonant enhancement of noise within a confined range of the polarization mode dispersion parameter. Such an enhancement manifests itself through resonance-like growth of gain fluctuations due to escape of the signal state of polarization from the "polarization trapping" state [3], threshold-like dropping of the Kramers length and the Hurst parameter. The average polarization state remains "localized" but its sensitivity to an input state of polarization vanishes with the growth of polarization mode dispersion parameter. In this sense, a Raman gain in the vicinity of stochastic antiresonance plays a role of a "depolarizer" reducing the polarization dependent gain.

\section{MODEL}

The model for analysis of fibre Raman amplifier with randomly varying birefringence is based on the results of [4,5]. Transition to the reference frame, in which the birefringence vector on the Poincaré sphere is $\boldsymbol{W}_{i}=\left(2 b_{i}, 0,0\right)$, results in representation corresponding to random wandering of $\boldsymbol{W}_{i}$ in horizontal plane and rotation of the unit signal $\mathbf{s}=\left(s_{1}, s_{2}, s_{3}\right)$ $(i=s)$ and pump $\mathbf{p}=\left(p_{1}, p_{2}, p_{3}\right)(i=p)$ Stokes vectors around the birefringence vector with the frequencies $b_{i}=2 \pi / L_{i}\left(L_{i}\right.$ is a polarization beat-length). Excluding of average scalar gain by the means of normalization of signal power to $G_{\text {ave }}=\exp \left(\int_{0}^{L} g P_{0}\left(z^{\prime}\right) / 2 d z^{\prime}-\alpha_{s} L\right)$ ( $L$ is a fibre length, $g$ is a Raman gain coefficient, $\alpha_{s}$ is a signal loss coefficient, $P_{0}(z)=P_{i n} \exp \left(-\alpha_{p} z\right)$ is a pump power, $\alpha_{p}$ is a pump loss coefficient, and $P_{i n}$ is an input pump power) results in the following set of stochastic differential equations describing an evolution of signal and pump states of polarization [5]:

$$
\begin{aligned}
& \frac{d s_{0}}{d z}=\frac{g}{2} P_{0}(z) x, \\
& \frac{d x}{d z}=\frac{g}{2} P_{0}(z) s_{0}-2\left(b_{p}-b_{s}\right)\left(p_{3} s_{2}-p_{2} s_{3}\right), \\
& \frac{d \mathbf{s}}{d z}=\frac{g}{2} P_{0}(z) s_{0} \mathbf{p}+2 b_{s}\left(\begin{array}{c}
0 \\
-s_{3} \\
s_{2}
\end{array}\right)+\beta\left(\begin{array}{c}
s_{2} \\
-s_{1} \\
0
\end{array}\right), \\
& \frac{d \mathbf{p}}{d z}=2 b_{p}\left(\begin{array}{c}
0 \\
-p_{3} \\
p_{2}
\end{array}\right)+\beta\left(\begin{array}{c}
p_{2} \\
-p_{1} \\
0
\end{array}\right), \\
& \frac{d \theta}{d z}=\beta(z) .
\end{aligned}
$$

Here $s_{0}$ is a signal power, $x=\mathbf{s} \cdot \mathbf{p}$ is a projection of signal state of polarization (SOP) to pump SOP, $\theta$ is a randomly varying 
angle between $\boldsymbol{W}$ and $X$-axis of the Poincare sphere. In the frameworks of fixed modulus model of random birefringence, the noise source $\beta(z)$ can be treated as a Wiener process with

$$
\langle\beta(z)\rangle=0,\left\langle\beta(z), \beta\left(z^{\prime}\right)\right\rangle=\sigma^{2} \delta\left(z-z^{\prime}\right),
$$

where $\langle\ldots\rangle$ means averaging over $z$, and $\sigma^{2}=1 / L_{c}\left(L_{c}\right.$ is a birefringence correlation length).

\section{Stochastic Anti-Resonance}

Stochastic differential equations $(1,2)$ were solved by direct numerical simulations as well as by applying the analytical multi-scale averaging procedure taking into account scales of both regular and random birefringence [5-7]. The dependences of normalized average gain coefficient $\langle G\rangle=10 \log \left(\left\langle s_{0}(L)\right\rangle / s_{0}(0)\right)$ and dispersion of its fluctuations $\sigma_{G}=\sqrt{\left\langle s_{0}^{2}(L)\right\rangle /\left\langle s_{0}(L)\right\rangle^{2}-1}$ on the polarization mode dispersion parameter $D_{p}$ [8] are shown in Figure 1. The numerical averaging was performed over ensemble of 100 independent stochastic trajectories.

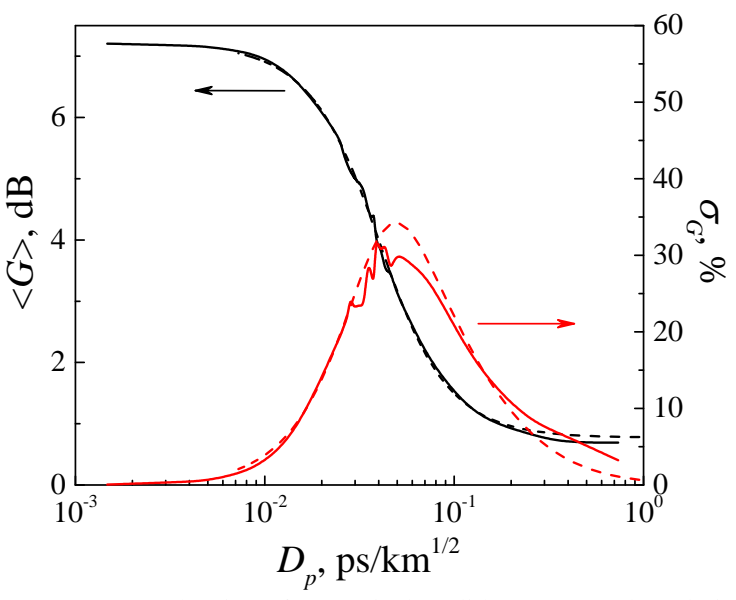

Figure 1. Dependencies of numerical (solid curves) and analytical (dashed curves) averaged gain $\langle G\rangle$ (black) and its dispersion $\sigma_{G}$

(red) on the PMD parameter $D_{p}$. The initial SOPs are $\mathbf{s}=(1,0,0), \mathbf{p}=$ $(1,0,0), s_{0}=10 \mathrm{~mW}, P_{\text {in }}=1 \mathrm{~W}$.

Figure 1 demonstrates a perfect agreement between the results of analytical multi-scale averaging technique and the direct numerical simulations of Equations $(1,2)$. Thus, cumbersome numerical simulations of long high-speed telecommunication lines can be replaced by comparatively simple analytical methods.

Figure 1 demonstrates an asymptotical behaviour of averaged gain for large and small PMDs. The maximal gain for $D_{p} \rightarrow 0\left(L_{i}>>L_{c}\right)$ and the minimum dispersions correspond to the Manakov's limit [3] when a single averaging scale coincides with $L_{c}$ :

$$
\frac{d \mathbf{S}}{d z}=\frac{g}{2}(\mathbf{P}|\mathbf{S}|+|\mathbf{P}| \mathbf{S})-\alpha_{s} \mathbf{S}+\left(b_{s}-b_{p}\right) \exp \left(-2 \sigma^{2} z\right)\left(\begin{array}{c}
0 \\
-S_{3} \\
S_{2}
\end{array}\right),
$$

$$
\frac{d \mathbf{P}}{d z}=-\frac{g \omega_{p}}{2 \omega_{s}}(\mathbf{P}|\mathbf{S}|+|\mathbf{P}| \mathbf{S})-\alpha_{p} \mathbf{P},
$$

where the pump depletion is taken into account, $\omega_{p}$ and $\omega_{s}$ are the pump and signal frequencies, respectively [9]. In this limit, a fibre behaves like an isotropic medium and the gain is defined by polarization pulling caused by attraction of the signal SOP to the pump SOP (i.e., $\langle x\rangle \rightarrow 1$ ) [3]. Such a phenomenon can be described as a trapping of randomly fluctuating particle in a potential well (Figure 2).

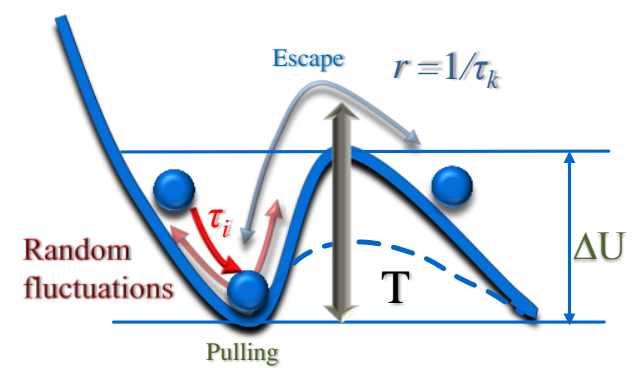

Figure 2. Potential well $\Delta U$ created by polarization pulling and modulated by birefringence with the period $\mathrm{T}$ defined by beatlength. Fluctuations of SOP caused by random birefringence are characterized by the relaxation length $\tau_{i}$ and by the escape rate $r=1$ / $\tau_{k}$ ( $\tau_{k}$ is a Kramers length).

Large PMDs $\left(L_{i}<<L_{c}\right)$ result in minimum gain and dispersion (Figure 1) as the pump and signal SOPs are almost decorrelated due to fast polarization beatings (i.e., $\langle x\rangle \rightarrow 0$ ). A "particle" is out potential well that is it escapes from the polarization pulling state (Figure 2). A fibre resembles the polarization maintaining one with rare "kicks" induced by random birefringence. Raman gain is defined by averaged pump SOP and is close to the scalar gain $G_{a v e}$ [3].

The most interesting phenomenon appears in the intermedium region of $D_{p} \approx 10^{-2} \div 10^{-1} \mathrm{ps} / \mathrm{km}^{1 / 2}$. The gain fluctuations (Figure 1) and the rate of escape from potential well increase abruptly [4,6]. The last corresponds to the threshold-like drop of the Kramers length [10] in Figure 3. Simultaneously, the Hurst parameter characterizing a longscale memory in a system [11] decreases from 1 (persistent statistics) for small PMDs and approaches the Brownian limit of $H=1 / 2$ (Figure 3 ). This noise-induced intensification of escape from metastable (polarization pulling) state [12] can be interpreted as a stochastic anti-resonance. The noise enhancement distinguishes this phenomenon from the stochastic resonance for which the relative intensity noise is suppressed [1,2]. Nevertheless, there is no complete chaotization in the case of stochastic anti-resonance because the Hurst parameter $0.9>H>1 / 2$ (Figure 3 ) that is it remains in the region of persistent statistic.

\section{CONCLUSION}

It was shown both numerically and analytically, that a fibre Raman amplifier with randomly varying birefringence demonstrates a resonant-like enhancement of gain fluctuations within diapason of PMDs corresponding to modern telecommunication systems. Such an enhancement can be 
interpreted as a stochastic anti-resonance and is characterized by abrupt growth of gain dispersion as well as by threshold-like drop of the Kramers length and the Hurst parameter. As was demonstrated analytically, the stochastic anti-resonance is multi-scaling phenomenon and develops when the scales of polarization beat-lengths and correlation length of random birefringence become commensurable. The results obtained provide with a new insight into multi-scaling nature of stochastic phenomena in the periodically driven systems and are usable for simulations of real-world high-speed communication lines.

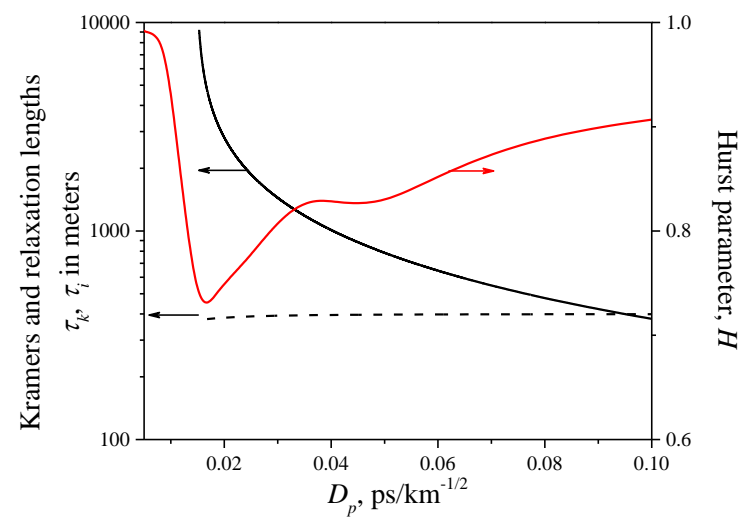

Figure 3. The Kramers (solid black curve) and relaxation (dashed black curve) lengths as well as the Hurst parameter (solid red curve) vs. the PMD parameter $D_{p}$.

\section{ACKNOWLEDGMENTS}

Support of the FP7-PEOPLE-2012-IAPP (project GRIFFON, No. 324391) is acknowledged. The computational results have been achieved using the Vienna Scientific Cluster (VSC).

\section{REFERENCES}

[1] B. Lindner, J. Garsia-Ojalvo, and L. Schimansky-Greif, "Effects of noise in excitable systems," Phys. Reports, vol. 392, pp. 321-424, 2004.

[2] L. Gammaitoni, P. Hänggi, P. Jung, and F. Marchesoni, "Stochastic resonance,” Rev. Mod. Phys. vol. 70, pp. 223-287, 1998.

[3] V. V. Kozlov, J. Nuño, J. D. Ania-Castañón, and S. Wabnitz, “Trapping polarization of light in nonlinear optical fibers: an ideal Raman polarizer," in Spontaneous Symmetry Breaking, Self-trapping, and Josephson Oscillations. Progress in Optical Science and Photonics, B. A. Malomed, Ed. Berlin: Springer, 2012, pp. 227-246.

[4] Q. Lin, G. P. Agrawal, "Vector theory of stimulated Raman scattering and its application to fiber-based Raman amplifiers," J. Opt. Soc. Am. B, vol. 20, pp. 1616-1631 (2003)

[5] S. Sergeyev, S. Popov, A. T. Friberg, "Modeling polarization dependent gain in fiber Raman amplifiers with randomly varying birefringence," Optics Commun. vol. 262, pp. 114-119 (2006).

[6] S. Sergeyev, "Activated polarization pulling and de-correlation of signal and pump states of polarization in fiber raman amplifier," Opt. Express, vol. 19, pp. $24268-24279$ (2011).

[7] S. Sergeyev, S. Popov, A.T. Friberg, "Virtually isotropic transmission media with fiber Raman amplifier," IEEE J. Quantum Electron. vol QE46, pp. 1492-1497 (2010).

[8] A. Galtarossa, C. R. Menyak, Polarization Mode Dispersion, Eds. NewYork: Springer, 2005.

[9] V. Kalashnikov, S. V. Sergeyev, G. Jacobsen, S. Popov, S. K. Turitsyn, "Multi-scale polarisation phenomena," Light: Science \& Applications (to be published).

[10] B. Lindner, J. Garsia-Ojalvo, A. Neiman, L. Schimansky-Greif, "Effects of noise in excitable systems," Phys Reports, vol. 392, pp. 321-424 (2004).

[11] J . Gao, Y. Cao, W. Tung, J. Hu, Multiscale Analysis of Complex Time Series: Integration of Chaos and Random Fractal Theory, and Beyond. New Jersey: Wiley-Blackwell, 2007.

[12] P. Hanggi, "Escape from a metastable state," J. Statistical Physics, vol. 42, pp. 105-148 (1986) 